\title{
Experimental Validations on Self Interference Cancelled Non-Orthogonal SEFDM Signals
}

\author{
Tongyang Xu and Izzat Darwazeh
}

\author{
Department of Electronic and Electrical Engineering, University College London, London, UK \\ Email: t.xu@ee.ucl.ac.uk, i.darwazeh@ucl.ac.uk
}

\begin{abstract}
Spectral efficiency can be improved in multicarrier systems through the employment of non-orthogonal overlapping sub-carriers, termed spectrally efficient frequency division multiplexing (SEFDM), but with self-created interference. Previous work has focused on signal detection development. The tradeoff between performance and complexity is challenging. This work investigates a self interference cancellation scheme for SEFDM to make use of ICI information at the transmitter and simplify the design of receiver. Repetition codes are used in the system where the same symbol with opposite signs are modulated onto adjacent sub-carriers. Therefore, ICI caused by adjacent sub-carriers would be cancelled mutually. However, the spectral efficiency is reduced. In order to maintain the same spectral efficiency and mutual interference cancellation benefits, the optimal combination of various modulation formats and bandwidth compression factors have to be studied jointly to derive maximum achievable spectral efficiency. Both simulation and experiment are reported and results validate the performance of the proposed self interference cancellation scheme.

Index Terms-Experiment, multicarrier communications, spectral efficiency, OFDM, SEFDM, non-orthogonal.
\end{abstract}

\section{INTRODUCTION}

The increase of data rate and a reduction of latency are equally important for future $5^{\text {th }}$ generation (5G) networks [1]. In order to improve data rates, SEFDM was developed in [2] where sub-carriers are non-orthogonally overlapped. Thus, occupying the same bandwidth, the data rate of SEFDM is higher than that of OFDM. However, the complicated signal processing at the receiver could add significant latency. To overcome the aforementioned challenge, two solutions are provided; one is to gather distributed complicated signal processing work together in a form of cloud-radio access networks (C-RAN) [3]; the other is to design new algorithms that can simplify receiver side signal processing. This work will investigate the second solution that signals would be precoded at the transmitter and relax the receiver side complexity. This is an efficient power saving technique for down-link transmission since a base station (BS) can handle complicated signal processing while the battery use in a mobile terminal is limited. In multiple input multiple output (MIMO) systems, linear precoding schemes based on channel inversion show the least complexity while the performance is far from the optimal one. Their non-linear substitutes such as dirty paper coding (DPC) [4], vector perturbation (VP) [5] and TomlinsonHarashima precoding (THP) [6] can significantly improve the performance but with higher complexity.
According to existing work [2], trying to extract useful information from distorted SEFDM signals would result in inaccurate signal estimate and increased error rate. Thus, any efforts at the receiver side seem to be difficult to improve system performance since ICI has been added to each subcarrier. Thereofre, an alternative solution is to mitigate the ICI at the beginning by precode signals before transmission at the transmitter side. Work in [7] has shown an singular value decomposition (SVD) based precoding system. However, signal detection is still limited by sophisticated signal processing.

This work proposed a self interference cancellation framework, which was firstly proposed in [8] for the purpose of mitigating the frequency offset in OFDM systems. An SEFDM signal has a similar effect of frequency offset since subcarriers are packed non-orthogonally intentionally. Therefore, the cancellation scheme would be applicable for SEFDM systems. The general principle of the cancellation scheme is to modulate the same symbol onto adjacent two sub-carriers (or a group of more than two sub-carriers) with opposite weighting coefficients (e.g. ' +1 ' and '-1') in order to minimize the ICI caused by the channel frequency errors (in SEFDM would be sub-carrier non-orthogonality packing). Thus, the ICI can be self cancelled at the transmitter. At the receiver, using a linear combination scheme, ICI could be further removed.

\section{BANDWIDTH SAVING WAVEFORM DESIGN}

The bandwidth compressed waveform has been comprehensively studied in [2] where the discrete signal of $N$ sub-carriers is expressed as

$$
X[k]=\frac{1}{\sqrt{Q}} \sum_{n=0}^{N-1} s_{n} \exp \left(\frac{j 2 \pi n k \alpha}{Q}\right)
$$

where $\alpha=\Delta f T$ is the bandwidth compression factor, which determines the bandwidth saving, $X[k]$ is the $k^{t h}$ time sample with index $k$ ranging from 0 to $Q-1, Q$ is the number of samples, which is defined as $Q=\rho N$ and $\rho \geq 1$ is the oversampling factor. The signal can further be simplified in a matrix format as

$$
X=\mathbf{F} S
$$

where $\mathbf{F}$ is the sub-carrier matrix with elements equal to $e^{\frac{-j 2 \pi n k \alpha}{Q}}$ and $S$ is the $N$-dimensional symbols.

The spectra in Fig. 1 show the bandwidth saving benefit of SEFDM and its challenge. As shown in the figure, SEFDM 


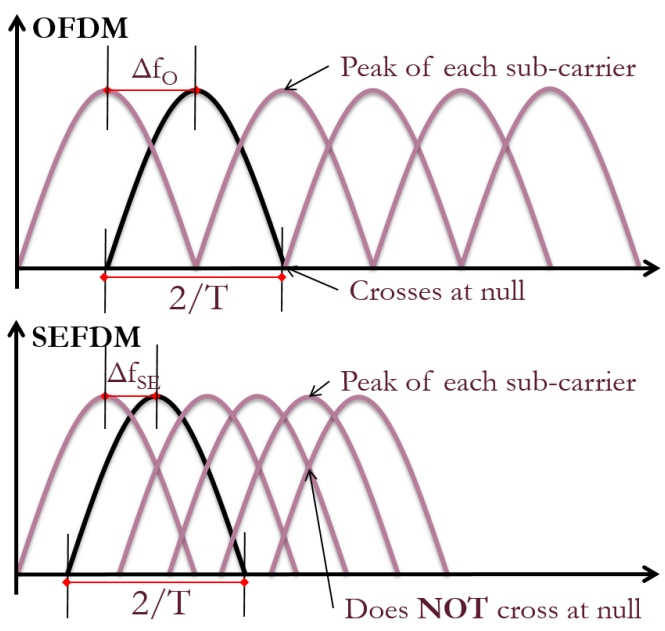

Figure 1. Spectra illustration for OFDM and SEFDM.

sub-carriers overlap with non-zero crossing point. Thus, selfcreated inter carrier interference (ICI) is introduced. The ICI has been fully studied in [2]. The receiver side signal is expressed as

$$
R=\mathbf{F}^{*} X+\mathbf{F}^{*} Z=\mathbf{F}^{*} \mathbf{F} S+\mathbf{F}^{*} Z=\mathbf{C} S+Z_{\mathbf{F}^{*}}
$$

where $R$ is an $N$-dimensional vector of demodulated symbols, $\mathbf{F}^{*}$ is the conjugate transpose of $\mathbf{F}$ and $Z$ is the additive white Gaussian noise (AWGN) vector. $\mathbf{C}$ is an $N \times N$ correlation matrix defined as $\mathbf{C}=\mathbf{F}^{*} \mathbf{F}$ with elements expressed as

$$
\mathbf{C}(m, n)=\frac{1}{Q} \times\left\{\begin{array}{ll}
Q & , m=n \\
\frac{1-e^{j 2 \pi \alpha(m-n)}}{1-e^{\frac{j 2 \pi \alpha(m-n)}{Q}}} & , m \neq n
\end{array}\right\}
$$

where the diagonal elements $(m=n)$ indicate auto-correlation values, which are all ones. The non-diagonal elements $(m \neq n)$ indicate cross-correlation, which in other words is the ICI. Thus, the following work aims to cancel the interference via a simple approach at the transmitter.

\section{SELF InTERFERENCE CANCELlation}

Following a technique similar to that in [8], proposed for OFDM, the basic idea is to arrange symbols as $S(1)=-S(0)$, $S(3)=-S(2), \ldots, S(N-1)=-S(N-2)$, thus the first demodulated symbol is given as

$$
R^{\prime}(0)=\sum_{k=0 \& k=\text { even }}^{N-2} S(k)[\mathbf{C}(0, k)-\mathbf{C}(0, k+1)]+Z_{\mathbf{F}^{*}}(0)
$$

where the correlation component in this case is expressed as

$$
\mathbf{C}^{\prime}(0, k)=\mathbf{C}(0, k)-\mathbf{C}(0, k+1)
$$

Therefore, carrier-to-interference power ratio (CIR) expressed here only for a single sub-carrier, is defined as

$$
C I R=\frac{|\mathbf{C}(0,0)-\mathbf{C}(0,1)|^{2}}{\sum_{k=2 \& k=\text { even }}^{N-2}|\mathbf{C}(0, k)-\mathbf{C}(0, k+1)|^{2}}
$$

In addition, the second demodulated symbol is defined in (8) with its correlation component in (9).

$$
R^{\prime}(1)=\sum_{k=0 \& k=\text { even }}^{N-2} S(k)[\mathbf{C}(1, k)-\mathbf{C}(1, k+1)]+Z_{\mathbf{F}^{*}}(1)
$$

$$
\mathbf{C}^{\prime}(1, k)=\mathbf{C}(1, k)-\mathbf{C}(1, k+1)
$$

The aforementioned ICI cancellation is operated at the transmitter and is noted as 'Tx-cancellation'. In order to improve further the performance, a joint transmitter and receiver ICI cancellation scheme, noted as 'Tx-Rx-cancellation', is proposed below. The solution, given a new demodulated signal $R^{\prime \prime}(0)$, is mathematically expressed as

$$
\begin{aligned}
R^{\prime \prime}(0)= & R^{\prime}(0)-R^{\prime}(1) \\
= & \sum_{\substack{k=0 \& k=\text { even }\\
}}^{N-2} S(k)[\mathbf{C}(0, k)-\mathbf{C}(0, k+1)- \\
& \mathbf{C}(1, k)+\mathbf{C}(1, k+1)]+Z_{\mathbf{F}^{*}}^{\prime}(0)
\end{aligned}
$$

where $Z_{\mathbf{F}^{*}}^{\prime}(0)=Z_{\mathbf{F}^{*}}(0)-Z_{\mathbf{F}^{*}}(1)$. Since $\mathbf{C}$ is a Toeplitz matrix, therefore $\mathbf{C}(0, k)=\mathbf{C}(1, k+1)$. A new expression is given as

$$
\begin{aligned}
R^{\prime \prime}(0)= & \sum_{\substack{k=0 \& k=\text { even } \\
\mathbf{C}(1, k)]+Z_{\mathbf{F}^{*}}^{\prime}(0)}}^{N-2} S(k)[2 \mathbf{C}(0, k)-\mathbf{C}(0, k+1)-
\end{aligned}
$$

where a new correlation component is $\mathbf{C}^{\prime \prime}(0, k)=2 \mathbf{C}(0, k)-$ $\mathbf{C}(0, k+1)-\mathbf{C}(1, k)$ and a new CIR expression is defined as

$$
C I R=\frac{|2 \mathbf{C}(0,0)-\mathbf{C}(0,1)-\mathbf{C}(1,0)|^{2}}{\sum_{k=2 \& k=\text { even }}^{N-2}|2 \mathbf{C}(0, k)-\mathbf{C}(0, k+1)-\mathbf{C}(1, k)|^{2}}
$$

Three correlation components have been studied so far. The comparisons of them are included in Fig. 2 and Fig. 3. Two different bandwidth compression factors such as $\alpha=0.8$ and $\alpha=0.6$ are tested and each figure includes three different self cancellation schemes. 


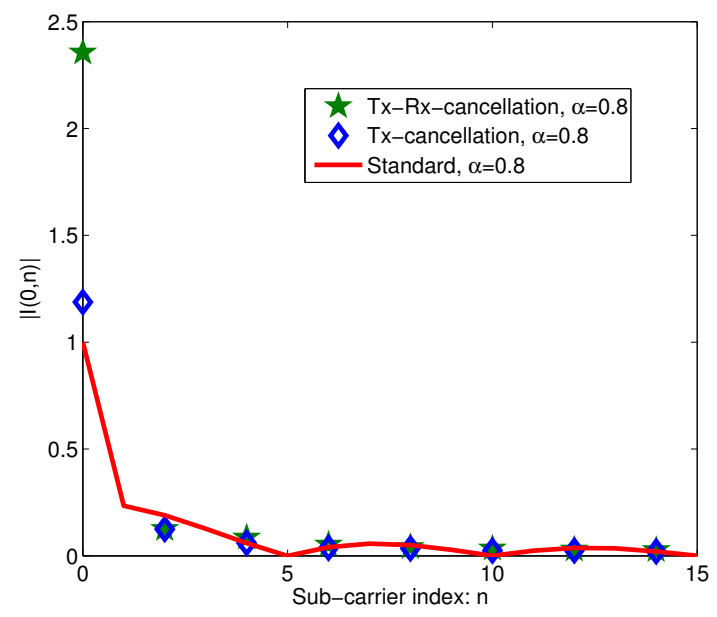

Figure 2. Comparisons of three systems in terms of desired and undesired power contributions to the first demodulated symbol $R(0)$ at $\alpha=0.8$.

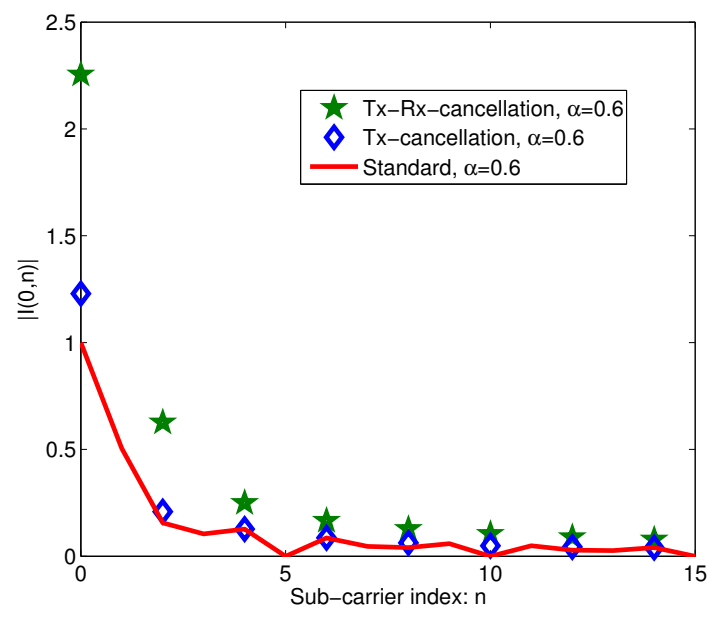

Figure 3. Comparisons of three systems in terms of desired and undesired power contributions to the first demodulated symbol $R(0)$ at $\alpha=0.6$.

Desired and undesired power distributions are studied in Fig. 2 and Fig. 3. The power values are based on correlation components such as $\mathbf{C}(0, k), \mathbf{C}^{\prime}(0, k)$ and $\mathbf{C}^{\prime \prime}(0, k)$. A general term, $\mathbf{I}(0, n)$, is therefore used instead of those. When $n=0$, $|\mathbf{I}(0, n)|$ indicates the desired signal while ICI components are conditioned by $n>0$.

In Fig. 2, it is evident that the Tx-Rx-cancellation scheme has the highest desired signal power, Tx-cancellation one is in the middle while the standard SEFDM achieves the lowest. In terms of ICI components, they all have similar ICI power. It should be noted that the Tx-Rx-cancellation scheme and the Tx-cancellation scheme only take even values, and the number of the interfering sub-carriers is reduced by $50 \%$ relative to the standard SEFDM case, leading to the improved CIR performance evident in Fig. 4.
In Fig. 3, a smaller bandwidth compression factor $\alpha$ is used. The desired signal power is the same as that of Fig. 2 while the ICI component power is different among three systems. It is apparent that the Tx-cancellation scheme shows a similar interference power with the standard one while the Tx-Rxcancellation scheme presents a higher interference power.

Finally, the desired and undesired power contributions are studied in the form of CIR. Both the Tx-cancellation and the Tx-Rx-cancellation schemes show improved CIR relative to the standard SEFDM one. The Tx-Rx-cancellation one offers more than two orders of magnitude CIR improvement from $\alpha=0.7$ to $\alpha=0.9$. This is due to the increased desired signal power as shown in Fig. 2. However, below $\alpha=0.7$, Txcancellation scheme starts to show a higher CIR. The reason may be inferred from Fig. 3 where the interference from the Tx-Rx-cancellation scheme is much higher than that of the Tx-cancellation SEFDM.

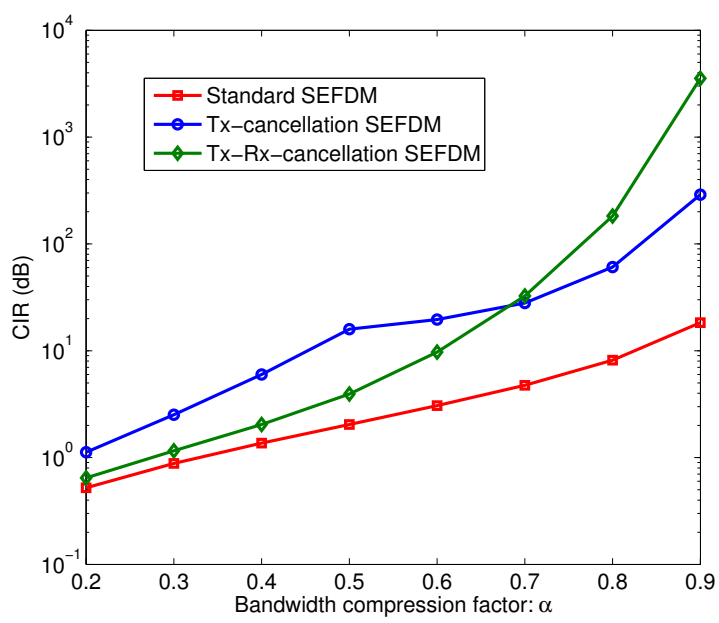

Figure 4. CIR comparisons for three systems with $\mathrm{N}=16$ sub-carriers over various bandwidth compression factors $\alpha$.

Constellation quality is used as an initial performance assessment of the self interference cancellation schemes. Fig. 5 shows 16QAM constellation patterns for SEFDM signals with $20 \%$ bandwidth compression (i.e. $\alpha=0.8$ ). Fig. 5(a) shows a typical constellation diagram with no interference cancellation. It is clearly seen that the constellation points scatter significantly due to the self-created ICI. It is expected that the signal associated with this constellation cannot be recovered easily. Fig. 5(b) presents a better constellation diagram due to the use of transmitter self interference cancellation. However, a fixed phase rotation of the constellation is observed. This can be compensated for at the receiver, which is shown in Fig. 5(c). The quality of the constellation is greatly improved because the scattering and the phase rotation have been compensated. The improved result also verifies the conclusion obtained from Fig. 4 where the CIR of the Tx-Rx-cancellation scheme is much higher than others at $\alpha=0.8$. In order to get further improved constellation performance, advanced soft demapping 


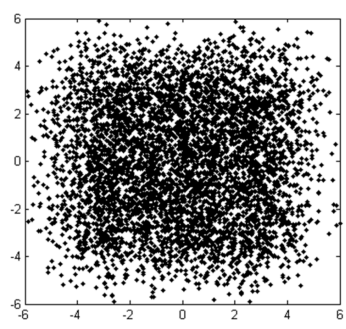

(a)

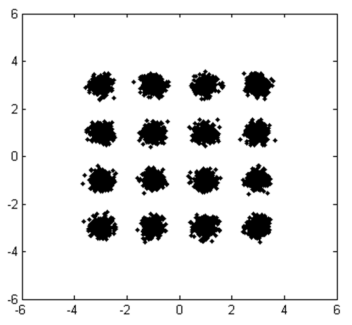

(c)

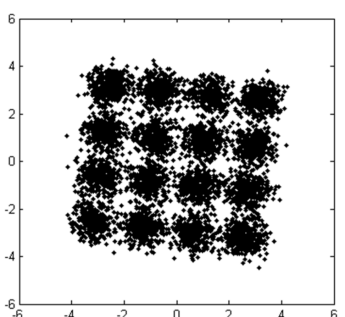

(b)

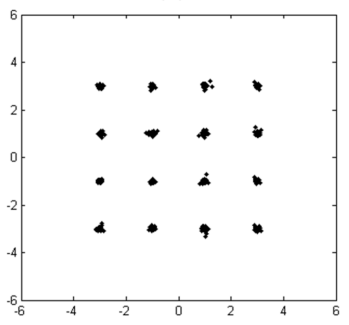

(d)
Figure 5. Constellation illustrations for 16QAM SEFDM with $\alpha=0.8$. (a) No cancellation; (b) Tx-cancellation; (c) Tx-Rx-cancellation; (d) Tx-Rx-ID cancellation.

derived from work in [9] is tested in Fig. 5(d) where a much better constellation pattern can be obtained.

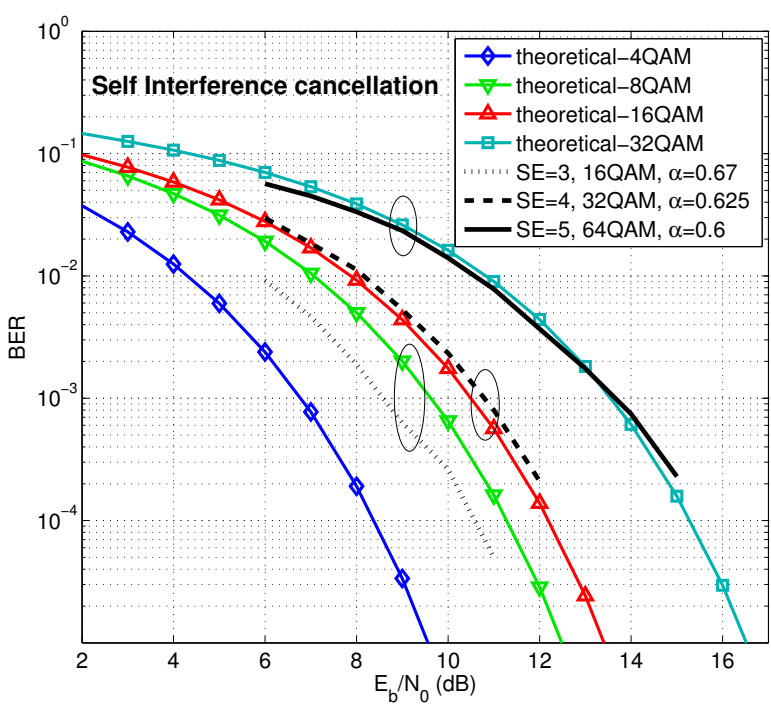

Figure 6. BER comparisons of self interference cancellation SEFDM systems and OFDM systems at different spectral efficiencies.

Fig. 6 presents the simulated results of SEFDM systems with Tx-Rx-cancellation in (11). OFDM systems are included for comparisons and are denoted as 'theoretical-MQAM'. For illustration, only a simple AWGN channel is considered here. Noting that a symbol is transmitted twice with opposite signs resulting in halved spectral efficiency, in order to have fair comparisons, systems with the same achievable spectral efficiency have to be compared. Thus, modulation formats
Table I

Achievable Spectral EFFiciency

\begin{tabular}{ll}
\hline Parameters & 16QAM \\
\hline \hline Raw spectral efficiency & $4 \mathrm{bit} / \mathrm{s} / \mathrm{Hz}$ \\
\hline Self-IC spectral efficiency & $2 \mathrm{bit} / \mathrm{s} / \mathrm{Hz}$ \\
\hline $\begin{array}{l}\text { Self-IC with SEFDM signals } \\
(33 \% \text { bandwidth saving) }\end{array}$ & $3 \mathrm{bit} / \mathrm{s} / \mathrm{Hz} \Longleftrightarrow 8 \mathrm{QAM}$ OFDM \\
\hline
\end{tabular}

and bandwidth compression factors should be flexible for SEFDM. One example of the comparable spectral efficiency for SEFDM is presented in Table I. It is clearly seen in Fig. 6 that SEFDM outperforms OFDM at spectral efficiency equals $3 \mathrm{bit} / \mathrm{s} / \mathrm{Hz}$. For other spectral efficiency values, SEFDM shows the same performance as that of OFDM. These results are expected since higher constellation density introduces higher interference, which is beyond the correction capability. With further bandwidth compression, further performance loss is expected.

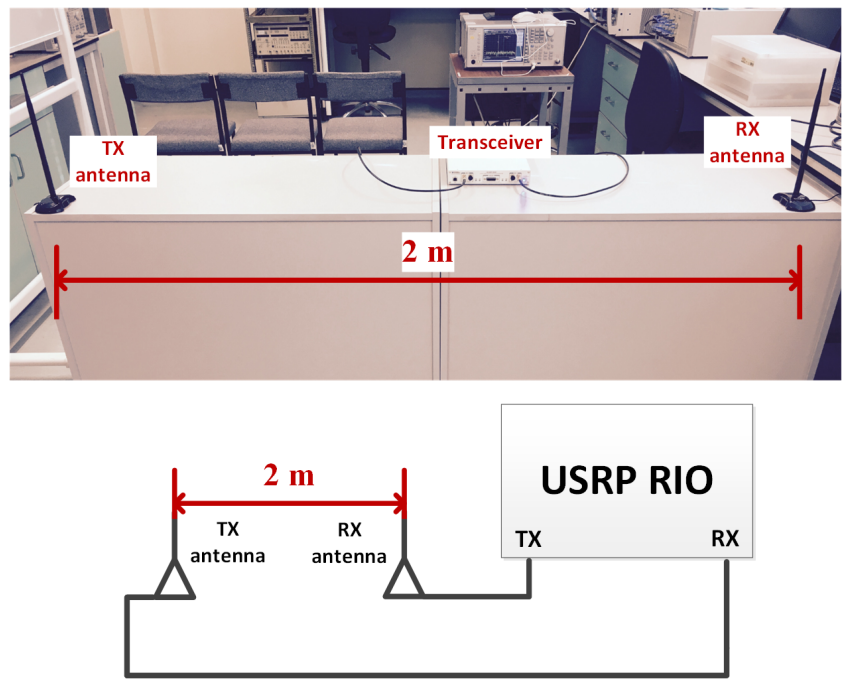

Figure 7. Single SEFDM/OFDM testbed setup using USRP RIO.

\section{OVER-THE-AIR INDOOR EXPERIMENT}

The experimental setup is designed on a single USRP based SEFDM/OFDM platform. The actual setup is shown in Fig. 7 where one USRP RIO 2953R [10] is used as a transceiver. The main benefit of this testbed is that the entire SEFDM system can be jointly implemented in software and hardware. LabVIEW is the software tool that implements part of the functions aligning with an FPGA chip integrated in the USRP RIO platform. A computer is connected to the USRP RIO and is used to generate digital signals to be transmitted and to process the received signals.

The analogue signal processing is integrated in the USRP RIO device. The wireless signal transmission link is set up via two omnidirectional antennas with a $8 \mathrm{dBi}$ peak gain in the azimuth plane. In order to extend the coverage of the transmit and receive antennas, a 5m RF coaxial cable with 
Table II

EXPERIMENTAL TESTBED SPECIFICATIONS For SINGLE USRP SySTEMS

\begin{tabular}{lll}
\hline Parameters & OFDM & SEFDM \\
\hline \hline Central carrier frequency $(\mathrm{GHz})$ & 2.4 & 2.4 \\
\hline Modulation scheme & 4QAM & 4QAM \\
\hline Sampling rate $(\mathrm{MHz})$ & 7.68 & 7.68 \\
\hline FFT size & 1024 & 1024 \\
\hline Number of data sub-carriers & 600 & 600 \\
\hline Bandwidth compression factor & 1 & 0.8 \\
\hline Sub-carrier spacing (kHz) & 15 & 12 \\
\hline Channel bandwidth $(\mathrm{MHz})$ & 5 & 4 \\
\hline Data bandwidth $(\mathrm{MHz})$ & 4.5 & 3.6 \\
\hline Data rate $(\mathrm{Mbps})$ & 9 & 9
\end{tabular}

negligible attenuation is connected between the Tx antenna (or Rx antenna) and the USRP RF0 out port (or RF1 in port). Two antennas are arranged with a line-of-sight (LOS) link and the spacing between the two antennas is $2 \mathrm{~m}$, with the antennas being placed $0.8 \mathrm{~m}$ above the floor. The experiment is operated according to the system specifications in Table. II where the central carrier frequency is set to the free license $2.4 \mathrm{GHz}$ and other parameters are mostly set based on LTE standard. The table indicates that transmitting the same number of bits per second, SEFDM signal $(\alpha=0.8,20 \%$ bandwidth saving) occupies a narrower bandwidth.

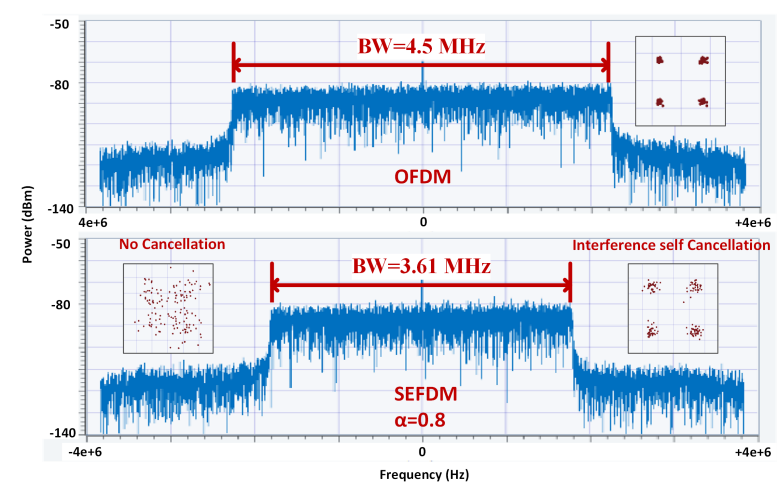

Figure 8. Baseband OFDM (top) and SEFDM (bottom) spectra on a single USRP RIO device.

Screenshots of the constellation performance, are studied and illustrated in the insets of Fig. 8. Firstly, a typical OFDM spectrum together with a constellation pattern, following LTE specifications, are presented. The occupied data bandwidth is 4.51 MHz, which follows the LTE requirement [11]. Second, an SEFDM signal with a bandwidth compression factor $\alpha=0.8$ is evaluated in Fig. 8. It is clearly seen that the occupied signal bandwidth is compressed. Without interference cancellation, the constellation points are more scattered. After using the self interference cancellation method, the constellation performance is similar to the OFDM one. It should be noted that the experimental results are not consistent with the simulation results observed in Fig. 6. The reason for this is due to the interference and noise from the USRP device and the indoor wireless channel. The compensation for those effects would be the future improvement work for the self interference cancellation SEFDM system.

\section{CONCLUSIONS}

This work reported low complexity SEFDM transceivers design. The scheme, termed self interference cancellation, aims to transmit the same symbols twice but with opposite signs, on adjacent sub-carriers. This solution has trade-off between complexity and spectral efficiency. Typical SEFDM signal detectors are too complicated due to multiplication and matrix inverse computations. However, the proposed cancellation scheme follows the typical OFDM operations where no matrix inversion is needed. It should be noted that using a group of two symbols, the spectral efficiency is halved. Therefore, achievable spectral efficiency is studied in this work. Results indicate that this solution is suitable for low order modulation formats. For achievable spectral efficiency equals $3 \mathrm{bit} / \mathrm{s} / \mathrm{Hz}$, SEFDM outperforms typical OFDM. For other achievable spectral efficiency values, SEFDM and OFDM have similar BER performance.

\section{ACKNOWLEDGEMENT}

This work was funded by the Engineering and Physical Sciences Research Council (EPSRC) "Discovery to Use Impact Acceleration" award for the development of a precommercialization $5 \mathrm{G}$ transceiver prototype. The work was also supported by National Instruments and by a generous donation of the LTE framework.

\section{REFERENCES}

[1] J. Andrews, S. Buzzi, W. Choi, S. Hanly, A. Lozano, A. Soong, and J. Zhang, "What will $5 \mathrm{G}$ be?" Selected Areas in Communications, IEEE Journal on, vol. 32, no. 6, pp. 1065-1082, June 2014.

[2] T. Xu and I. Darwazeh, "Transmission experiment of bandwidth compressed carrier aggregation in a realistic fading channel," IEEE Transactions on Vehicular Technology, vol. 66, no. 5, pp. 4087-4097, May 2017.

[3] A. Checko, H. L. Christiansen, Y. Yan, L. Scolari, G. Kardaras, M. S Berger, and L. Dittmann, "Cloud RAN for mobile networks-a technology overview," IEEE Communications Surveys Tutorials, vol. 17, no. 1, pp. 405-426, Firstquarter 2015.

[4] M. Costa, "Writing on dirty paper (Corresp.)," IEEE Transactions on Information Theory, vol. 29, no. 3, pp. 439-441, May 1983.

[5] J. Maurer, J. Jalden, D. Seethaler, and G. Matz, "Vector perturbation precoding revisited," IEEE Transactions on Signal Processing, vol. 59, no. 1, pp. 315-328, Jan 2011.

[6] C. Windpassinger, R. F. H. Fischer, T. Vencel, and J. B. Huber, "Precoding in multiantenna and multiuser communications," IEEE Transactions on Wireless Communications, vol. 3, no. 4, pp. 1305-1316, July 2004.

[7] S. Isam and I. Darwazeh, "Precoded spectrally efficient FDM system," in Personal Indoor and Mobile Radio Communications (PIMRC), 2010 IEEE 21st International Symposium on, Sept 2010, pp. 99-104.

[8] Y. Zhao and S.-G. Haggman, "Intercarrier interference self-cancellation scheme for OFDM mobile communication systems," Communications, IEEE Transactions on, vol. 49, no. 7, pp. 1185-1191, Jul 2001.

[9] T. Xu, R. C. Grammenos, F. Marvasti, and I. Darwazeh, "An improved fixed sphere decoder employing soft decision for the detection of nonorthogonal signals," Communications Letters, IEEE, vol. 17, no. 10, pp. 1964-1967, October 2013.

[10] National Instruments, "USRP-RIO-2953R, Software Defined Radio Reconfigurable Device," http://sine.ni.com/nips/cds/view/p/lang/en/nid/ 213005.

[11] 3GPP TS 36.300 version 8.12.0 Release 8, "Evolved universal terrestrial radio access (E-UTRA) and evolved universal terrestrial radio access network (E-UTRAN); overall description; stage 2 (release 8)," April 2010. 